\title{
Comportamento Sexual de Estudantes de um Curso de Medicina do Rio de Janeiro
}

\author{
Sexual Behavior of Medical Students in Rio de \\ Janeiro, Brazil
}

\author{
Júlio César Soares Aragão ${ }^{I}$ \\ Claudia de Souza Lopes ${ }^{I I}$ \\ Francisco Inácio Bastos ${ }^{I I I}$
}

\section{PALAVRAS-CHAVE: \\ - Sexualidade \\ - Uso de Preservativos \\ - Infecções Sexualmente \\ Transmissíveis \\ - Estudantes de Medicina}

\section{KEYWORDS:}

- Sexuality

- Condom Uses

- Sexually Transmitted Infections

- Medical Students
Recebido em: 03/02/2010

Reencaminhado em: 12/10/2010

Aprovado em: 10/02/2011

REVISTA BRASILEIRA DE EDUCAÇ̃̃O MÉDICA
${ }^{I}$ Centro Universitário de Volta Redonda, Volta Redonda, RI, Brasil.

"Universidade do Estado do Rio de Janeiro, RJ, Brasil.

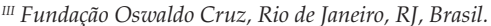

\section{RESUMO}

O uso consistente de preservativos constitui a principal estratégia de prevenção do HIV/aids, bem como de outras infecções sexualmente transmissíveis (ISTs). Neste contexto, o perfil de uso de preservativos em estudantes de Medicina é de especial interesse, principalmente quando consideramos o papel de formadores de opinião desempenhado pelos profissionais médicos. Este estudo investiga o padrão de comportamento sexual e sua associação com o uso de preservativos entre estudantes do curso de Medicina de uma universidade privada de um município do interior do Estado do Rio de Janeiro. Dados relativos a 643 estudantes de Medicina foram colhidos por meio de um questionário anônimo e autopreenchível. As análises foram conduzidas utilizando-se a regressão de Poisson com variância robusta para o cálculo das razões de prevalência brutas e ajustadas. A maioria dos alunos respondeu já ter iniciado vida sexual (85,3\%). O parceiro da primeira relação sexual foi, em sua maioria, o(a) namorado(a) (55,1\%), e o local da coitarca mais frequentemente relatado foi a própria casa $(27,1 \%)$. O método anticoncepcional mais frequentemente utilizado na coitarca foi o preservativo $(90,1 \%)$. $\mathrm{Na}$ análise bivariada, o fator que se mostrou mais estreitamente associado ao uso inconsistente do preservativo foi a presença de parceria estável, achado corroborado pela análise multivariada, após ajuste por sexo, idade e renda ( $R P=1,95$ IC 95\% 1,47 - 2,57). Estes achados são compatíveis com a hipótese de que indivíduos inseridos em parcerias estáveis têm menor percepção de risco por suporem que a estabilidade de seus relacionamentos thes conferiria proteção relativa contra as ISTs e HIV/aids.

Consistent condom use is still a key component in the prevention of HIV/AIDS and other sexually transmitted infections. In this context, of particular interest are condom use patterns by medical students, especially considering the role of the latter as opinion-makers. The present article reports data on sexual behavior and its association with condom use among medical students in a private medical school in Rio de Janeiro State, Brazil. Data were collected from 643 medical students using an anonymous, self-administered questionnaire. Data analysis used Poisson regression with robust variance, calculating crude and adjusted prevalence ratios. Most students were sexually initiated (85.3\%). The most common partners in their sexual debut were their boyfriends/girlfriends $(55.1 \%)$, most often in their own homes (27.0\%). Condoms were the main contraceptive method used at sexual debut (90.1\%). In bivariate analysis, sex with a stable partner was the main factor associated with inconsistent condom use, and this association was confirmed by multivariate analyses, adjusted for age, gender, and family income ( $P R=1.95 ; 95 \%$ CI 1.47-2.57). These findings are consistent with the hypothesis that individuals with stable partnerships are less likely to perceive themselves at risk, following the supposition that the stability of the relationship protects against HIV/AIDS and other STIs. 


\section{INTRODUÇÃO}

O século XX foi um período de revoluções em todas as áreas do relacionamento e desenvolvimento humano. A sociedade experimentou modificações substanciais, e a sexualidade foi uma das áreas mais profundamente transformadas no âmbito da cultura ocidental. As mudanças nas relações de gênero, a introdução de métodos anticoncepcionais seguros e eficazes, o controle e tratamento das infecções sexualmente transmissíveis (ISTs) bacterianas (como a gonorreia e a sífilis), entre tantas outras mudanças, trouxeram à tona uma sexualidade mais aflorada, a ser vivida e experimentada de forma diversa da vivência sexual construída sob os padrões de repressão vigentes nos moldes da era vitoriana ${ }^{1}$

As profundas mudanças no âmbito dos comportamentos sexuais tiveram impacto importante sobre as ISTs, o que, junto com a emergência do HIV/aids na década de 1980, conferiu nova ênfase aos métodos de prevenção. Dentre as diferentes formas de profilaxia das ISTs, o uso do preservativo de látex masculino, camisa de vênus ou camisinha, tem sido o mais propalado por várias agências de saúde, governamentais ou não, com elevado grau de proteção documentado frente ao risco de aquisição de infecções sexualmente transmissíveis (ISTs), inclusive o HIV/aids, por diferentes revisões sistemáticas e meta-análises (ver, por exemplo, Holmes et al. ${ }^{2}$ ).

Segundo o Programa Conjunto das Nações Unidas em Aids (Unaids) ${ }^{3}$, o uso do preservativo em todas as relações sexuais é o mais simples e eficiente método disponível para a prevenção da transmissão sexual do HIV e demais ISTs. Relativamente barato (referimo-nos ao preservativo masculino, pois o feminino é substancialmente mais caro), com pouca ou nenhuma restrição ao uso, pode ser utilizado com segurança, sem exigir qualquer habilidade específica (também aqui, com relação ao preservativo feminino, a colocação exige maior prática e familiaridade), sendo eficaz como método contraceptivo e na prevenção de outras infecções/doenças sexualmente transmissíveis ${ }^{4,5}$.

De acordo com Spizzichino et al. ${ }^{6}$, as infecções entre os jovens constituem uma importante parcela das novas infecções por HIV na população mundial, sendo este segmento populacional considerado uma das populações-alvo para ações de educação e prevenção ${ }^{3}$. Em nosso país, o quadro descrito nas estatísticas globais se repete: dos 362.364 casos de aids citados anteriormente, 33.867 (9,4\%) foram diagnosticados em jovens com idades entre 20 e 24 anos $^{7}$. O quadro referente às demais ISTs é, basicamente, similar, embora os dados sejam bastante mais incompletos.

Embora os alunos de cursos universitários constituam apenas uma pequena parcela de nossa população jovem, é importante ressaltar seu papel como formadores de opinião, $\mathrm{o}$ que define estes jovens como um grupo-chave ao se formularem estratégias de educação e prevenção da infecção pelo HIV e demais ISTs ${ }^{8}$. Dentre o restrito grupo formado pelos universitários, os estudantes de Medicina são de especial interesse, não só pelo conhecimento específico a que estão normalmente expostos, mas também por virem a estar futuramente envolvidos com a prevenção da aids e demais IDTs, e pela eventual assistência a pessoas acometidas por estas afecções em sua prática profissional ${ }^{9}$.

O consenso atual é que a prevenção primária da transmissão do HIV e de grande parte das ISTs se baseia principalmente na prática de formas de sexo consideradas mais seguras. As principais formas do sexo seguro consideradas válidas para a prevenção, até o presente momento, são: uso consistente (em todas as relações) do preservativo masculino de látex ${ }^{10}$; uso do preservativo feminino, utilizado de forma semelhante ao masculino; atividades sexuais em que não haja troca de fluidos orgânicos (práticas sem penetração); e redução do número de parceiros sexuais, associada à postergação ou abstenção de relações sexuais ${ }^{11}$.

A adesão ao uso do preservativo tem sido amplamente documentada em várias publicações, em âmbito tanto nacional como internacional. Todavia, ainda é grande a demanda por estudos que possibilitem conhecer em maior detalhe os hábitos de diferentes estratos populacionais em relação às práticas de sexo seguro, especialmente nos países em desenvolvimento.

O principal objetivo do presente estudo foi avaliar o padrão de comportamento sexual e sua associação com o uso de preservativos entre estudantes de um curso de Medicina.

\section{MÉTODOS}

\section{Desenho de Estudo e População de Referência}

Trata-se de um estudo seccional, do tipo censo, realizado em estudantes regularmente inscritos e com matrícula ativa no curso de Medicina de uma universidade privada em um município do interior do Estado do Rio de Janeiro. A instituição contava, em agosto de 2006, com 5.227 alunos, sendo 724 alunos matriculados no curso de Medicina e distribuídos em 12 períodos semestrais. Todos os alunos do curso de Medicina foram convidados a participar do estudo.

\section{Medidas}

Foi utilizado como instrumento de avaliação um questionário autopreenchível, que incluía questões relativas a aspectos sociodemográficos e econômicos, coitarca (primeira relação sexual) e comportamento sexual.

O uso de preservativo (desfecho em estudo) foi avaliado pela Escala de Estágios de Mudança para Uso de Preserva- 
tivo - Stages of Change for Preservative Use Scale (SCCUS) - adaptada para o português por Meyer et al. ${ }^{12}$ Os estágios da escala são classificados em: Pré-Contemplação, Contemplação, Preparação, Ação e Manutenção. O uso foi definido como "consistente", uma vez que o indivíduo se apresentasse na fase de manutenção da escala, tendo sido o desfecho avaliado de forma dicotômica.

As variáveis de exposição relativas ao comportamento sexual investigadas neste estudo foram: coitarca, orientação sexual, atividade sexual, parceiro na coitarca, local da coitarca, uso de contraceptivos na coitarca, tipo de contraceptivo usado na coitarca, prescrição de contraceptivos na coitarca (quem prescreveu), ter (ou não) parceiro fixo e ter (ou não) relacionamentos afetivo-sexuais ocasionais. As categorias utilizadas em cada variável estão descritas na Tabela 1 .

As covariáveis consideradas no estudo foram: (a) características sociodemográficas e econômicas: sexo, idade, ciclo do curso, raça (autorrelatada), religião de criação, religião atual, estado civil, escolaridade do pai, escolaridade da mãe e renda familiar per capita; (b) primeira menstruação ou ejaculação; (c) ter (ou não) filhos; (d) gravidez (Tabela 2).

O questionário foi apresentado aos alunos no ambiente escolar, de acordo com as atividades regulares agendadas para cada período, evitando ocupar horários que prejudicassem suas atividades de ensino. O preenchimento do questionário foi realizado após a leitura e assinatura do Termo de Consentimento Livre e Esclarecido por parte dos alunos. Os questionários foram preenchidos de forma anônima, garantindo que os dados referentes a determinado aluno não pudessem ser identificados. Após o preenchimento do Termo de Consentimento Informado e do questionário, o próprio aluno o depositava numa urna lacrada, que somente era aberta ao final da coleta dos dados. O estudo foi aprovado pelo Comitê de Ética da Universidade no qual o estudo foi conduzido.

\section{Análise Estatística}

Numa primeira etapa, foram geradas e examinadas as distribuições de frequência de cada variável que integrava o instrumento utilizado no estudo. Em seguida, foram realizadas análises bivariadas para avaliar a associação entre as variáveis em estudo e o uso consistente de preservativo. Utilizamos o teste qui-quadrado para verificar se as associações encontradas apresentavam significância estatística $(\mathrm{p}<0,10)$, valendo-nos deste critério para a seleção das possíveis variáveis de confusão. Aquelas associadas tanto à exposição quanto ao desfecho foram testadas por meio de modelos multivariados. Na análise multivariada, utilizamos as razões de prevalências brutas e ajustadas, e seus respectivos intervalos de 95\% de confiança, calculadas através de Modelo Linear Generalizado por regressão de Poisson com variância robusta, conforme proposto por Barros e Hirakata ${ }^{14}$. O modelo de ajuste incluiu as variáveis: "sexo", "idade" e "renda familiar per capita".

A análise dos dados foi realizada com auxílio do programa Stata, versão 8 .

\section{Resultados}

Entre os 724 estudantes matriculados no curso de Medicina, $643(88,8 \%)$ preencheram e retornaram os questionários do estudo. A frequência de respostas variou de questão para questão. Todavia, a pequena proporção de respostas não preenchidas não comprometeu a análise de nenhuma questão em especial (dados não apresentados).

A idade dos alunos variou entre 17 e 43 anos, com valor médio de 22 anos. A maioria dos alunos se classificou como da raça branca $(76,0 \%)$. Perguntados sobre sua religião atual, a maioria se definiu como católica $(57,5 \%)$. O estado civil predominante foi solteiro $(97,0 \%)$. Outras informações sobre os aspectos socioeconômicos se encontram na Tabela 1.

A primeira menstruação/ejaculação ocorreu, em média, aos 12,2 anos (IC 95\% 12,1 - 12,3). A média de idade na coitarca foi de 16,5 anos (IC 95\% 16,4 - 16,7). A maioria dos alunos afirmou ser heterossexual $(98,4 \%)$ e já ter iniciado vida sexual (85,3\%). O parceiro da primeira relação sexual foi o/a namorado(a) na maioria dos casos $(55,1 \%)$, e o local da coitarca mais frequentemente relatado foi a própria casa do respondente $(27,1 \%)$. Algum método anticoncepcional foi empregado na coitarca em $85,0 \%$ dos casos, sendo o preservativo o mais frequente $(90,1 \%)$. A prescrição de método anticoncepcional por um médico ocorreu somente em $20,9 \%$ dos estudantes.

Mais da metade $(62,2 \%)$ dos estudantes referiu ter parceiro fixo e $47,2 \%$ afirmaram ter relacionamentos afetivo-sexuais ocasionais. Dois terços $(74,5 \%)$ dos entrevistados relataram ter tido atividade sexual nos 30 dias anteriores à pesquisa, e, adicionalmente, $10,6 \%$ relataram ter tido atividade sexual considerado um período mais dilatado, de 60 dias. Apenas 14,9\% dos entrevistados referiram não ter tido nenhuma atividade sexual nos últimos 60 dias. O número de parceiros sexuais (no período de até 60 dias anteriores à aplicação do questionário) entre os estudantes que referiram atividade sexual variou entre um e dez parceiros. A atividade sexual com parceiro único foi relatada pela maioria $(72,5 \%)$ dos respondentes, enquanto a atividade sexual com dois parceiros foi relatada por $15,4 \%$ dos estudantes.

Quanto ao uso de preservativos, a partir da utilização da classificação da escala SCCUS, verificou-se que 29,7\% dos respondentes foram classificados no estágio de pré-contempla- 
Tabela 1 - Características sócio-demográficas em estudantes de Medicina de uma Universidade privada no interior do Estado do Rjo de Janeiro $(n=643)$.

\begin{tabular}{|c|c|c|}
\hline Tabela 1 - Características $\mathrm{s}$ & $n$ & $\%$ \\
\hline \multicolumn{3}{|l|}{ Sexo } \\
\hline Masculino & 322 & 50,1 \\
\hline Femino & 321 & 49,9 \\
\hline \multicolumn{3}{|l|}{ Ciclo do Curso } \\
\hline Básico & 221 & 34,4 \\
\hline Clínico & 210 & 32,7 \\
\hline Internato & 212 & 33,0 \\
\hline \multicolumn{3}{|l|}{ Idade } \\
\hline Até 20 anos & 189 & 30,4 \\
\hline 21 a 25 anos & 357 & 57,5 \\
\hline 26 anos ou mais & 75 & 12,1 \\
\hline \multicolumn{3}{|l|}{ Raça } \\
\hline Pardos e Negros & 109 & 17,3 \\
\hline Brancos & 488 & 77,3 \\
\hline Outras & 34 & 5,4 \\
\hline \multicolumn{3}{|l|}{ Religião de Criação } \\
\hline Católica & 467 & 72,6 \\
\hline Não-Católica & 176 & 27,4 \\
\hline \multicolumn{3}{|l|}{ Religião Atual } \\
\hline Católica & 370 & 57,5 \\
\hline Nâo-Católica & 273 & 42,5 \\
\hline \multicolumn{3}{|l|}{ Estado Civil } \\
\hline Casado & 16 & 2,5 \\
\hline Solteiro & 624 & 97,1 \\
\hline Divorciado & 3 & 0,5 \\
\hline \multicolumn{3}{|l|}{ Escolaridade do $\mathrm{Pai}$} \\
\hline Fundamental Incompleto & 30 & 4,7 \\
\hline Fundamental Completo & 36 & 5,6 \\
\hline Médio & 156 & 24,3 \\
\hline Superior & 418 & 65,0 \\
\hline Nẫo conheci & 3 & 0,5 \\
\hline \multicolumn{3}{|l|}{ Escolaridade da Mãe } \\
\hline Fundamental Incompleto & 18 & 2,8 \\
\hline Fundamental Completo & 47 & 7,3 \\
\hline Médio & 190 & 29,6 \\
\hline Superior & 388 & 60,3 \\
\hline Nấo conheci & 0 & 0,0 \\
\hline \multicolumn{3}{|l|}{ Renda Familiar per capita } \\
\hline Até $\mathrm{R} \$ 1.500$ & 189 & 30,4 \\
\hline Entre $\mathrm{R} \$ 1501$ a $\mathrm{R} \$ 3000$ & 357 & 57,5 \\
\hline Acima de $R \$ 3001$ & 75 & 12,1 \\
\hline
\end{tabular}

ção, contemplação em 15,5\%, preparação em 6,5\%, ação em $3,9 \%$ e $44,5 \%$ foram classificados como inseridos no estágio de manutenção. A estratificação por gênero mostrou haver diferenças estatisticamente significativas entre homens e mulheres com relação a coitarca precoce, atividade sexual atual, parceria da coitarca, local e uso de contraceptivos na coitarca, parceria fixa e relacionamentos esporádicos (Tabela 2). Quanto ao período cursado, não houve diferença estatisticamente significan-

\begin{tabular}{|c|c|c|c|c|c|c|c|}
\hline \multirow[t]{2}{*}{ Variável } & \multicolumn{2}{|c|}{ Gera } & \multicolumn{2}{|c|}{ Feninino } & \multicolumn{2}{|c|}{ Masculino } & \multirow[b]{2}{*}{$p$} \\
\hline & $n$ & $\%$ & $n$ & $\%$ & $n$ & $\%$ & \\
\hline \multicolumn{8}{|l|}{$\begin{array}{l}\text { Primeira menstruaçăo ou } \\
\text { Ejaculaçäo }\end{array}$} \\
\hline Abaixo de 10 anos & 74 & 11.6 & 31 & 9.7 & 43 & 13.7 & \\
\hline Com 11 anos ou mas & 562 & 88.6 & 290 & 90.3 & 272 & 86.3 & 0.116 \\
\hline \multicolumn{8}{|l|}{ Coitarca } \\
\hline Abaixo de 15 anos & 79 & 14.9 & 10 & 4.4 & 69 & 22.8 & \\
\hline Com 15 anos ou mas & 212 & 33.0 & 217 & 95.6 & 234 & 77.2 & $<0,001$ \\
\hline \multicolumn{8}{|l|}{ Oriertação Sexual } \\
\hline Heterossexual & 632 & 98.4 & 318 & 98.9 & 314 & 98.1 & \\
\hline Honossexual & 3 & 0.5 & 2 & 0.6 & 1 & 0.3 & \\
\hline Bissexual & 7 & 1.1 & 2 & 0.6 & 5 & 1.6 & 0.441 \\
\hline \multicolumn{8}{|l|}{ Atividade sexual } \\
\hline $\operatorname{sim}$ & 547 & 85.3 & 235 & 73.2 & 312 & 97.5 & \\
\hline Nẵo & 94 & 14.7 & $\infty$ & 26.8 & 8 & 2.5 & $<0,001$ \\
\hline \multicolumn{8}{|l|}{ Parceiro da coitarca } \\
\hline Namcrado & 301 & 55.1 & 200 & 85.1 & 101 & 32.5 & \\
\hline "Ficante" & 149 & 27.3 & 18 & 7.7 & 131 & 42.1 & \\
\hline Amigo & 29 & 5.3 & 9 & 3.8 & 20 & 6.4 & \\
\hline Empregada(o) & 13 & 2.4 & 0 & 0.0 & 13 & 4.2 & \\
\hline Profissional do sexo & 36 & 6.6 & 1 & 0.4 & 35 & 11.3 & \\
\hline Outro & 18 & 3.3 & 7 & 3.0 & 11 & 3.5 & $<0,001$ \\
\hline \multicolumn{8}{|l|}{ Local da Coitarca } \\
\hline Casa & 148 & 27.1 & 54 & 22.9 & 94 & 30.2 & \\
\hline Hotel ou Motel & 81 & 14.8 & 42 & 17.8 & 39 & 12.5 & \\
\hline Praia & 19 & 3.5 & 6 & 2.5 & 13 & 4.8 & \\
\hline Casa de Amigos & 68 & 13.4 & 17 & 7.2 & 51 & 16.4 & \\
\hline Casa do parceiro & 104 & 19.0 & 70 & 29.7 & 34 & 10.9 & \\
\hline Carro & 34 & 6.2 & 8 & 3.4 & 26 & 6.2 & \\
\hline Casa de Campo & 17 & 3.1 & 8 & 3.4 & 9 & 28.9 & \\
\hline República & 24 & 4.4 & 21 & 8.9 & 3 & 1.0 & \\
\hline Outros & 52 & 9.5 & 10 & 4.2 & 42 & 13.5 & $<0,001$ \\
\hline \multicolumn{8}{|c|}{ Usou contraceptivo na coitarca } \\
\hline $\operatorname{sim}$ & 463 & 85.0 & 210 & 89.7 & 253 & 81.4 & \\
\hline Nầo & 82 & 15.1 & 24 & 10.3 & 58 & 18.6 & 0,007 \\
\hline \multicolumn{8}{|l|}{$\begin{array}{l}\text { Tipo de contraceptivo } \\
\text { na coitarca }\end{array}$} \\
\hline Tabela & 10 & 2.1 & 5 & 2.3 & 5 & 1.9 & \\
\hline Camisirha & 429 & 90.1 & 178 & 83.2 & 251 & 95.8 & \\
\hline Coito interrompico & 5 & 1.1 & 4 & 1.9 & 1 & 0.4 & \\
\hline Pílua & 30 & 6.3 & 25 & 11.7 & 5 & 1.9 & \\
\hline Camisirha Femirina & 2 & 0.4 & 2 & 0.9 & 0 & 0.0 & $<0,001$ \\
\hline \multicolumn{8}{|c|}{$\begin{array}{l}\text { Prescriçäo de contraceptivo } \\
\text { na coitarca }\end{array}$} \\
\hline Opróprio & 325 & 68.6 & 102 & 47.9 & 223 & 85.4 & \\
\hline Médico & 99 & 20.9 & 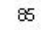 & 39.9 & 14 & 5.4 & \\
\hline Amigo & 7 & 1.5 & 1 & 0.5 & 6 & 2.3 & \\
\hline Parceiro & 31 & 6.5 & 24 & 11.3 & 7 & 2.7 & \\
\hline Outros & 12 & 2.5 & 1 & 0.5 & 11 & 4.2 & $<0,001$ \\
\hline \multicolumn{8}{|l|}{ Parceiro fixo } \\
\hline $\operatorname{sim}$ & 206 & 37.8 & 74 & 31.6 & 132 & 42.4 & \\
\hline & 339 & 62.2 & 160 & 68.4 & 179 & 57.6 & 0.01 \\
\hline \multicolumn{8}{|c|}{ Relacionamentos eqporádicos } \\
\hline $\operatorname{sim}$ & 287 & 52.9 & 170 & 72.7 & 117 & 37.9 & \\
\hline Nă̄o & 256 & 47.2 & 64 & 27.4 & 192 & 62.1 & $<0,001$ \\
\hline Uso consistente do con & & & & & & & \\
\hline Pré-contemplaçẵo & 138 & 29.7 & 62 & 32.1 & 76 & 27.9 & \\
\hline Cortemplaçäo & 72 & 15.5 & 30 & 15.5 & 42 & 15.4 & \\
\hline Preparaçắo & 30 & 6.5 & 10 & 5.2 & 20 & 7.4 & \\
\hline Açấo & 18 & 3.9 & 9 & 4.7 & 9 & 3.310 & \\
\hline Manutencão & 207 & 44.5 & 82 & 42.5 & 125 & 45.960 & 0.675 \\
\hline
\end{tabular}

te no uso do preservativo em diferentes períodos ou mesmo entre os ciclos básico, clínico e internato.

$\mathrm{Na}$ análise bivariada, observou-se que o uso consistente do preservativo se mostrou associado de forma estatisticamente significante a renda familiar per capita, parceria fixa e relacionamentos ocasionais (Tabela 3).

Na análise multivariada (Tabela 4), após ajuste por sexo, idade e renda familiar per capita, estudantes que relataram ter parceiro fixo apresentavam prevalência quase duas vezes maior de uso inconsistente de preservativo, se comparados àqueles sem parceiro fixo ( $\mathrm{RP}=1,95 \mathrm{IC} 95 \% 1,47-2,57)$. $\mathrm{O}$ relato de relacionamentos ocasionais, ao contrário, se mostrou associado de forma inversa com o uso inconsistente de preservativos $(R P=0,81$ IC $95 \% 0,67-0,97)$. 
Tabela 3 - Uso do condom em estudantes de Medicina de u ma Universidade privada no interior do Estado do Rio de Janeiro, segundo variáveis socioeconômicas e comportamento sexual

\begin{tabular}{|c|c|c|c|c|c|}
\hline \multirow[b]{3}{*}{ Variável } & \multicolumn{4}{|c|}{ Uso Consistente do Condom } & \multirow[b]{3}{*}{$\mathrm{P}$} \\
\hline & \multicolumn{2}{|c|}{ Sim } & \multicolumn{2}{|c|}{ กล๊ั } & \\
\hline & $n$ & $\%$ & $n$ & $\%$ & \\
\hline \multicolumn{6}{|l|}{$\operatorname{sex} 0$} \\
\hline Masculino & 82 & 42.5 & 111 & 57.5 & \\
\hline Femino & 125 & 45.9 & 147 & 54.0 & 0.458 \\
\hline \multicolumn{6}{|l|}{ Cido do Curso } \\
\hline Básico & 66 & 49.6 & 67 & 50.4 & \\
\hline Clínico & 65 & 38.7 & 103 & 61.3 & \\
\hline Internato & 76 & 46.3 & 8 & 53.7 & 0.140 \\
\hline \multicolumn{6}{|l|}{ Idade } \\
\hline Até 20 an $0 s$ & 55 & 52.9 & 49 & 47.1 & \\
\hline 21 a 25 ancs & 120 & 42.9 & 160 & 57.1 & \\
\hline 26 anos ou mais & 26 & 41.9 & 36 & 58.1 & 0.188 \\
\hline \multicolumn{6}{|l|}{ Raca } \\
\hline Pardos e Negros & 38 & 45.8 & 45 & 54.2 & \\
\hline Brancos & 155 & 43.9 & 198 & 56.1 & \\
\hline Outras & 13 & 54.2 & 11 & 45.8 & 0.607 \\
\hline \multicolumn{6}{|l|}{ Religi ق̃o de Criaçäo } \\
\hline Católica & 154 & 46.2 & 187 & 54.8 & \\
\hline Nâo-C atólica & 53 & 42.7 & 71 & 57.3 & 0.842 \\
\hline \multicolumn{6}{|l|}{ Religi כొ Atua } \\
\hline Católica & 121 & 45.8 & 143 & 54.2 & \\
\hline Não-C atólica & 86 & 42.8 & 115 & 57.2 & 0.512 \\
\hline \multicolumn{6}{|l|}{ Estado Civil } \\
\hline Casado & 5 & 31.3 & 11 & 68.8 & \\
\hline Solteiro/Dhrorciado & 202 & 45.0 & 247 & 55.0 & 0277 \\
\hline \multicolumn{6}{|l|}{ Ezoolaridade do Pai } \\
\hline Fund amental Incompleto & 6 & 21.4 & 22 & 78.6 & \\
\hline Fundamental Completo & 8 & 33.3 & 16 & 66.7 & \\
\hline Médio & 56 & 46.7 & 64 & 53.3 & \\
\hline Superior & 136 & 45.9 & 154 & 53.1 & \\
\hline Nắo conheci & 1 & 33.3 & 2 & 66.7 & 0.081 \\
\hline \multicolumn{6}{|l|}{ Escol aridade da Mö̀ } \\
\hline Fund amental Incompleto & 2 & 14.3 & 12 & 85.7 & \\
\hline Fundamental Completo & 16 & 50.0 & 16 & 50.0 & \\
\hline Médio & 61 & 43.9 & 78 & 56.1 & \\
\hline Superior & 128 & 45.7 & 152 & 54.3 & 0.124 \\
\hline Renda Familiar per capita & & & & & \\
\hline Até $R \$ 1.500$ & 63 & 3.7 & 100 & 61.3 & \\
\hline Entre $R \$ 1501$ a $R \$ 3000$ & 55 & 41.0 & 79 & 59.0 & \\
\hline Acima de $\mathrm{R} \$ 3001$ & 68 & 56.2 & 53 & 43.8 & 0.008 \\
\hline Primeira menstruação ou & & & & & \\
\hline 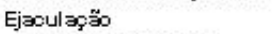 & & & & & \\
\hline Abako de 10 anos & 27 & 47.4 & 30 & 52.6 & \\
\hline Com 11 anos ou mais & 177 & 43.9 & 226 & 56.1 & 0.624 \\
\hline Coitarca & & & & & \\
\hline Abaixo de 15 anos & 28 & 36.4 & 49 & 63.6 & \\
\hline Com 15 anos ou mais & 174 & 45.5 & 200 & 53.5 & 0.103 \\
\hline Orientoç̃ Sexua & & & & & \\
\hline Heterossexual & 204 & 44.7 & 252 & 55.3 & \\
\hline Homossexual & 0 & 0.0 & 2 & 100.0 & \\
\hline Bissexual & 3 & 50.0 & 3 & 50.0 & 0.431 \\
\hline Usou contraceptivo na coit & & & & & \\
\hline Sim & 179 & 45.1 & 209 & 53.9 & \\
\hline Não & 28 & 37.3 & 47 & 62.7 & 0.161 \\
\hline Tipo de contraceptivo na co & & & & & \\
\hline Camisinha & 168 & 45.5 & 193 & 53.5 & \\
\hline Outros & 16 & 42.1 & 22 & 57.9 & 0.602 \\
\hline Parceiro fixo & & & & & \\
\hline Sim & 87 & 65.4 & 48 & 34.6 & \\
\hline Nâo & 119 & 36.1 & 211 & 63.9 & $<0,001$ \\
\hline Relacionamentos exporádic & & & & & \\
\hline Sim & 91 & 38.6 & 145 & 61.4 & \\
\hline Não & 115 & 50.9 & 111 & 49.1 & 0.008 \\
\hline
\end{tabular}

\section{Conclusões}

Embora o presente estudo se restrinja a dados relativos a um único momento de um segmento específico da população
Tabela 4 - Razões de Prevalência para ausência de uso consistente do preservativo em estudantes de Medicina de uma Universidade privada no interior do Estado do Rio de Janeiro, segundo parceria fixa e Relacionamentos ocasionais, ajustados por Sexo, Idade e Renda per capita familiar

\begin{tabular}{|c|c|c|c|c|}
\hline \multirow{2}{*}{ PARCEIRO FIXO } & \multirow{3}{*}{$\begin{array}{l}\mathrm{RP} \\
\mathbf{1 . 8 5}\end{array}$} & \multicolumn{3}{|c|}{$\mathrm{IC}(95 \%)$} \\
\hline & & & & \\
\hline & & 1.44 & - & 2.37 \\
\hline \multicolumn{5}{|c|}{ Modelo Ajustado por Sexo } \\
\hline & 1.86 & 1.45 & - & 2.39 \\
\hline \multicolumn{5}{|c|}{ Modelo Ajustado por Sexo e Idade } \\
\hline & 1.87 & 1.45 & - & 2.43 \\
\hline \multicolumn{5}{|c|}{ Modelo Ajustado por Sexo, Idade e Renda Familiar } \\
\hline & 1.95 & 1.47 & - & 2.57 \\
\hline \multicolumn{5}{|c|}{ RELACIONAMENTOS OCASIONAIS } \\
\hline & 0.80 & 0.68 & - & 0.94 \\
\hline \multicolumn{5}{|c|}{ Modelo Ajustado por Sexo } \\
\hline & 0.80 & 0.67 & - & 0.95 \\
\hline \multicolumn{5}{|c|}{ Modelo Ajustado por Sexo e Idade } \\
\hline & 0.79 & 0.66 & - & 0.94 \\
\hline \multicolumn{5}{|c|}{ Modelo Ajustado por Sexo, Idade e Renda Familiar } \\
\hline & 0.81 & 0.67 & - & 0.97 \\
\hline
\end{tabular}

(universitários do curso de Medicina), acreditamos que os dados ora expostos capturam um momento de um processo de importantes transformações no âmbito do comportamento sexual dos jovens brasileiros no tocante à primeira relação sexual e ao comportamento sexual de modo geral. Tais mudanças foram evidenciadas em estudos anteriores realizados no Brasil ${ }^{15,16}$, que documentam maior prevalência da primeira relação sexual com um parceiro estável e maior prevalência do uso consistente do preservativo, tanto na coitarca quanto nas relações sexuais subsequentes ${ }^{17}$. Possivelmente, estes dados refletem uma mudança cujas raízes se encontram no fato de estes jovens terem crescido em um ambiente em que a preocupação com sexo seguro e a percepção do risco têm presença ampla e constante.

A idade da primeira ejaculação/menstruação se mostrou similar àquela descrita por Bruno et al. ${ }^{18}$, que identificaram a média de 12 anos com relação a estes eventos no Estado de São Paulo.

A prevalência de uso consistente de preservativos encontrada $(44,5 \%)$, embora não seja ideal, é semelhante àquela verificada em outros segmentos populacionais - como os conscritos do Exército brasileiro, em um estudo de $2002^{19}$ - e em diferentes grupos sociais do município de Salvador (BA), no ano de $2000^{20}$, cujas taxas de uso consistente de preservativos oscilavam em torno de $40 \%$, embora Viana et al. ${ }^{21}$ tenham encontrado cifras em torno de $60 \%$, em estudantes do ensino médio no Estado de Minas Gerais. Esperava-se que em alunos de um curso médico esses números se apresentassem mais altos, refletindo maior percepção de risco por parte de futuros profissionais de saúde. 
A variável mais estreitamente associada ao uso inconsistente de preservativos no presente estudo foi a inserção do entrevistado em parceria estável, o que possivelmente está relacionado à percepção, nem sempre acurada, de menor risco por parte dos indivíduos inseridos em relacionamentos dessa natureza. Na verdade, o risco de se infectar através do sexo desprotegido é determinado pelo fato de haver ou não infecção pelo HIV em um dos dois parceiros e ainda em função da carga viral do parceiro eventualmente infectado. No caso de um parceiro com serostatus ignorado (situação bastante frequente), os riscos associados ao sexo desprotegido com parceiro estável podem ser maiores ou menores (se comparados ao sexo desprotegido com parceiros ocasionais de serostatus igualmente ignorado), dependendo do serostatus efetivo dos diferentes parceiros. Já no caso de sexo com um parceiro infectado pelo HIV, o uso consistente de preservativos é mandatório. Este achado é compatível com o exposto por Sanches ${ }^{22}$, que afirma que parcerias fixas podem resultar em um falso sentimento de "proteção". Pessoas que não têm relacionamentos estáveis tendem a se sentir mais vulneráveis às ISTs e, portanto, a se preocupar mais em evitar a infecção pelo HIV e demais ISTs, como assinalam Geluda et al. ${ }^{23}$ em estudo qualitativo conduzido com adolescentes na cidade do Rio de Janeiro. A mesma conclusão foi assinalada por Carreno e Costa ${ }^{24} \mathrm{em}$ um estudo populacional no interior do Rio Grande do Sul, em que mulheres com parceiros fixos, principalmente as casadas, mais frequentemente deixavam de utilizar os preservativos. A associação entre "estar inserida em relacionamento estável" e o uso inconsistente de preservativos se manteve, no referido estudo, após o ajuste por diferentes variáveis de confusão.

A presença de relacionamentos ocasionais (que não excluem a concomitância de um relacionamento estável) se mostrou associada ao uso consistente do preservativo, corroborando a hipótese de que estes indivíduos teriam uma percepção de maior risco em suas atividades sexuais, conforme indicado por Macaluso et al. ${ }^{25}$, em virtude de, potencialmente, se perceberem mais expostos na sua inter-relação com parceiros com quem têm menos intimidade.

Em um estudo de base nacional nos Estados Unidos, Finer et al. ${ }^{26}$ reportaram achados similares: mulheres com mais de um parceiro sexual referiam uso mais frequente dos preservativos. Estes achados reforçam a conclusão de Thomsen et al. ${ }^{27}$, que veem na percepção do risco o principal fator associado ao uso consistente do preservativo.

Com base nos achados do presente trabalho, cremos que a ênfase na percepção do risco dos indivíduos deva ser considerada, tanto ou mais que a informação adequada ${ }^{28}$, na formulação de políticas de saúde que favoreçam a adoção do uso de preservativos e a adesão dos jovens ao uso do preservativo em todas as suas relações sexuais. Esta estratégia tem sido frequentemente relegada a um segundo plano na formulação de risco, sendo a grande maioria de estratégias baseada simplesmente no caráter informativo de conteúdos e dados biológicos que não necessariamente dão ao jovem a vivência ou percepção do risco.

A principal limitação do presente estudo se deve ao fato de lidar com uma população restrita, com características peculiares (estudantes de Medicina de uma universidade privada). Outra limitação se deve ao fato de ter como objeto uma população que (a princípio) tem bom conhecimento teórico acerca da importância do uso do preservativo na prevenção das ISTs, fato que poderia levar a algumas discrepâncias nos dados obtidos, pois os estudantes poderiam ter optado pela resposta "mais correta" e relatado um uso de preservativo mais consistente do que aquele que de fato praticam, gerando subestimação do risco. Um estudo mais aprofundado desta população, com abordagens qualitativas, poderia propiciar uma visão complementar da situação, possibilitando a triangulação dos achados e maior aprofundamento e melhor compreensão dos mecanismos envolvidos.

Os dados do presente trabalho nos levam a refletir sobre a necessidade de avaliar (e preencher) algumas possíveis lacunas de formação de opinião baseadas nos ensinamentos teóricos do currículo atual dos estudantes de Medicina. Cremos que novas abordagens e ferramentas de ensino, baseadas na percepção de risco, possam agir sinergicamente no sentido de induzir vivências que propiciem um comportamento sexual mais seguro não somente em estudantes, mas na população como um todo.

\section{AGRADECIMENTOS}

Agradecemos a todos os alunos que participaram deste trabalho, por sua cooperação e acolhimento.

\section{REFERÊNCIAS}

1. Gay P. Education of the Senses: The Bourgeois Experience, Victoria to Freud [S1]: W. W. Norton \& Company;1999. v.1

2. Holmes KK, Levine R, Weaver M. Effectiveness of condoms in preventing sexually transmitted infections. Bull World Health Organ. 2004;82(6):454-61.

3. United Nations Programme on HIV/AIDS. Intensifying HIV prevention: a UNAIDS policy position paper. UNAIDS: Switzerland; 2005.

4. United Nations Programme on HIV/AIDS. Condom Social marketing: Selected case studies. UNAIDS: Switzerland; 2000. 
5. Centers for Disease Control. Update: Barrier Protection Against HIV infection and Other Sexually Transmitted Diseases. MMWR. 1993;42(30):589-91.

6. Spizzichino L, Piccinno F, Pedone G, Gallo P, Valli R, Cotti L, Bianconi M, Luzi AM. Adolescenti e HIV: Le campagne informative dei giovani per i Giovanni. Ann Ist Super Sanita. 2005;41(1):113-8.

7. Brasil. Ministério da Saúde. Boletim Epidemiológico Aids e DST. 2004;1(1):26-9.

8. Frauches DO, Rodrigues MMP. Estudantes de medicina e suas atitudes em relacäo à Aids em Vitória (ES). J Bras Psiquiatr. 2003;52(3):201-10.

9. Wang L, Zhang KL. AIDS-related knowledge, condom usage among medical postgraduates. Biomed Environ Sci. 2002;15(2):97-102.

10. Brasil. Ministério da Saúde. Manual de Controle das Doenças Sexualmente Transmissíveis — DST. Brasília:MS;2006

11. Vieira EM, Villela WV, Rea MF. Alguns aspectos do comportamento sexual e prática de sexo seguro em homens do Município de São Paulo. Cad Saúde Pública. 2000;16(4):997-1009.

12. Meyer E, Carvalhal A, Pechanskya F. Adaptation for Brazilian Portuguese of a scale to measure willingness to wear condoms. Rev Bras Psiquiatr. 2003;25(4):224-7.

13. Thompson ML, Myers JE, Kriebel D. Prevalence odds ratio or prevalence ratio in the analysis of cross sectional data: what is to be done? Occup Environ Med. 1998;55:272-7.

14. Barros AJD, Hirakata VN. Alternatives for logistic regression in cross-sectional studies: na empirical comparison of models that directly estimate the prevalence ratio. BMC BMC Med Res Methodol. 2003;3:21-34.

15. Borges ALV, Schor N. Início da vida sexual na adolescência e relações de gênero: um estudo transversal em São Paulo, Brasil, 2002. Cad Saúde Pública. 2005;21(2):499-507.

16. Pirotta KCM. Näo há guarda chuva contra o amor: estudo do comportamento reprodutivo e de seu universo simbólico entre jovens universitários da USP. São Paulo; 2002. Doutorado [Tese] — Universidade de Säo Paulo.

17. Heilborn ML, Aquino EML, Bozon L, Riva M, Knauth DR. O Aprendizado da Sexualidade. Rio de Janeiro: Garamond; 2006.

18. Bruno ZVS, Teixeira MA, Silva LGM, Silva RB, Guanabara RB, Oliveira EM, Chagas F. Sexualidade e anticoncepcäo na adolescência: conhecimento e atitude. Reprodução e Climatério. 1997;12(3):3.

19. Brasil. Ministério da Saúde. Programa Nacional de DST e Aids. Pesquisa entre conscritos do Exército Brasileiro -
Retratos do comportamento de risco do jovem brasileiro à infecção pelo HIV 1996 — 2002. Brasília: MS; 2006.

20. Codes JS, Cohen DA, Melo NA. Detecção de doenças sexualmente transmissíveis em ambientes clínicos e não-clínicos na Cidade de Salvador, Bahia, Brasil. Rev Bras Ginecol Obstet. 2002;24(2):101-6.

21. Viana FJM, Fagundes A, Mello MB. Fatores associados a sexo seguro entre alunos de escolas públicas em Minas Gerais, Brasil. Cad Saúde Pública. 2007;23(1):43-51.

22. Sanches KRB. A Aids e as Mulheres Jovens: Uma Questão de Vulnerabilidade. Rio de Janeiro; 1999. Doutorado [Tese] - Fundação Oswaldo Cruz.

23. Geluda K, Bosi MLM, Cunha AJLA, Trajman A. “Quando um não quer, dois não brigam": um estudo sobre o não uso constante de preservativo masculino por adolescentes do Município do Rio de Janeiro, Brasil. Cad Saúde Pública. 2006;22(8):1671-80

24. Carreno I, Costa JSD. Uso de preservativos nas relações sexuais: estudo de base populacional. Rev Saúde Pública. 2006;40(4):720-6.

25. Macaluso M, Demand MJ, Artz LM, Hook EW. Partner type and condom use. AIDS. 2000;14(5):537-46.

26. Finer LB, Darroch JE, Singh S. Sexual Partnership Patterns as a Behavioral Risk Factor For Sexually Transmitted Diseases. Fam Plann Perspectives. 1999;31(5):228-36

27. Thomsen S, Stalker M, Toroitich - Ruto C. Fifty ways to leave your rubber: how men in Mombasa rationalise unsafe sex. Sex Transm Infect. 2004;80:430-34.

28. Wingood GM, Diclemente RJ. HIV sexual risk reduction interventions for women: a review. Am J Prev Med. 1996;12(3):209-17.

\section{CONTRIBUIÇÃO DOS AUTORES}

Júlio César Soares Aragão participou do planejamento, coleta e análise de dados e na redação do manuscrito. Claudia de Souza Lopes e Francisco Inácio Bastos participaram do planejamento, análise de dados e redação do manuscrito.

\section{CONFLITO DE INTERESSES}

Declarou não haver.

\section{ENDEREÇO PARA CORRESPONDÊNCIA}

Júlio César Soares Aragão

Rua 156, 380 — sala 208

Laranjal - Volta Redonda

CEP. 27255-080 RJ

E-mail: julio.aragao@uol.com.br 